\title{
Structures of Inclusions of Impurity Nickel Atoms in Silicon Monocrystals
}

\author{
Turgunov Nozimjon Abdumannopovich, Berkinov Elmurod Khoshimjonovich
}

\begin{abstract}
Morphological parameters of microinclusions and nanoinclusions of impurity nickel atoms in silicon are studied by electron probe microscope. The dependence of the structural constructions of impurity clusters on their size and shape was studied. Quantitative indicators of the distribution of the atoms of the main and technological impurities over the volume of impurity clusters are established.

Keywords: accumulations of impurity nickel atoms, morphological parameters, electron probe microanalysis.
\end{abstract}

\section{INTRODUCTION}

Investigations of the processes occurring in the volume of semiconductor materials upon the introduction of impurities into them, the laws of their transformation, during which their composition and structure changes, is of particular interest from the point of view of determining the sequence of stages of formation of various accumulations of impurity atoms [1-4]. In this direction, the study of the nature of phenomena accompanying structural changes in matter, establishing the causes and methods of controlling physicochemical processes that ultimately determine the components and structural constructions of impurity clusters are most relevant. The identification of these factors is essential in the production of semiconductor materials with predetermined properties.

Currently, many leading laboratories and scientific centers of the world are conducting numerous experiments in the field of the dependence of the structure of accumulations of impurity atoms on its morphological parameters. In this regard, scanning probe microscopy is worth attention, which allows one to determine the quantitative ratios of impurity atoms and the main matrix in the volume of impurity clusters with fairly high accuracy (for metals it is $0.01 \%$ ).

The results of our previous studies showed that during diffusion doping of silicon single crystals with cobalt atoms, defective structures are formed in the form of microinclusions or nanoinclusions [5-7].

Revised Manuscript Received on April 18, 2020.

* Correspondence Author

Turgunov Nozimjon Abdumannopovich*, scientific secretary, Research Institute of Semiconductor Physics and Microelectronics at the National University of Uzbekistan, E-mail: tna_1975@mail.ru

Berkinov Elmurod Khoshimjonovich, doctoral student, Research Institute of Semiconductor Physics and Microelectronics at the National University of Uzbekistan.

(C) The Authors. Published by Blue Eyes Intelligence Engineering and Sciences Publication (BEIESP). This is an open access article under the CC BY-NC-ND license (http://creativecommons.org/licenses/by-nc-nd/4.0/)
It was shown that in the formation of impurity clusters, in addition to the atoms of the main impurity, atoms of uncontrolled impurities, such as copper, iron, chromium, which have relatively high diffusion and solubility coefficients, also take an active part. This paper presents the results of studies of the structural constructions and chemical composition of accumulations of nickel impurity atoms in silicon, as well as the influence of size and geometric shape on the structural constructions of microinclusions and nanoinclusions of impurity atoms.

\section{MATERIALS AND METHODS}

For research, we prepared samples of $\mathrm{Si}<\mathrm{Ni}>$, n- and ptype, obtained on the basis of the starting material monocrystalline silicon of the KEF brand, with specific resistances $\rho=5 \mathrm{Om} \cdot \mathrm{cm}$ and $\rho=20 \mathrm{Om} \cdot \mathrm{cm}$, respectively, grown by the Czochralski method. Nickel diffusion in silicon for both $\mathrm{n}-\mathrm{Si}<\mathrm{Ni}>$ and $\mathrm{p}-\mathrm{Si}<\mathrm{Ni}>$ samples was carried out at a temperature of $1523 \mathrm{~K}$ for 2 hours, after which the samples were subjected to slow and fast cooling. In the first case, the cooling rate ( $v$ cool) of the samples was $v_{\text {cool }} \leq 1 \mathrm{~K} / \mathrm{s}$. And with rapid cooling, it reached $v_{\text {cool }}=100$ $\mathrm{K} / \mathrm{s}$. The method of electron probe analysis, using the Superprobe JXA-8800R setup, carried out structural studies of impurity microinclusions and nanoinclusions. In terms of its sensitivity and accuracy, this setup is superior to other analogues and allows one to determine with high accuracy the chemical composition of individual sections of impurity clusters, the sizes of which are 100-150 nm.

\section{RESULTS AND DISCUSSION}

The results of experimental studies have shown that the morphology of microinclusions and nanoinclusions of impurity nickel atoms formed in the silicon bulk mainly depends on the value of $v_{\text {cool }}$ of the samples after diffusion annealing. Figure 1 shows images of accumulations of impurity atoms in $\mathrm{n}-\mathrm{Si}<\mathrm{Ni}>$ samples obtained with a slow (a) and fast (b) cooling rate. As can be seen in the volume of n-Si $<\mathrm{Ni}>$ samples obtained with slow cooling, impurity clusters with sizes ranging from several hundred nanometers to $\sim 5 \times 10^{-6} \mathrm{~m}$ are formed. The resulting clusters of impurity atoms, with relatively small sizes up to $\sim 800 \mathrm{~nm}$, i.e. impurity nanoinclusions have a needle-shaped, diskshaped, lenticular and spherical shape. In contrast, larger impurity clusters, the sizes of which reach up to several micrometers, i.e. impurity microinclusions have mainly a convex lenticular or spherical shape,

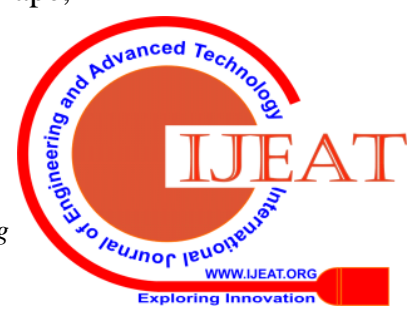

Sciences Publication

(C) Copyright: All rights reserved. 
as well as a complex multifaceted shape with a multilayer structure, the boundaries of which are clearly distinguished (Fig. 1 (a)). Depending on the shape and the size of multilayer microinclusions, each layer may have different thicknesses.

The density of such microinclusions according to the volume of the samples is on average $\sim 10^{2} \mathrm{~cm}^{-3}$.

In the volume of $\mathrm{n}-\mathrm{Si}<\mathrm{Ni}>$ samples obtained with $v_{\text {cool }}=$ $100 \mathrm{~K} / \mathrm{s}$, the formation of mainly referring to small clusters of impurity atoms is observed, the sizes of which reach up to 700-800 nanometers, and their average density is $\sim 2 \cdot 10^{3}$ $\mathrm{cm}^{-3}$. Such nanoinclusions have needle-shaped, disk-shaped, lenticular, and spherical shapes (Fig. 1 (b)). Lenticular clusters, in turn, can have biconvex, plano-convex, planoconcave or convex-concave forms (Fig. 1 (b)).

The results of the analysis of the chemical composition of the impurity microinclusion with a spherical shape, diameter $\mathrm{d}=1.9 \cdot 10^{-6} \mathrm{~m}$, which has a three-layer structure are shown in Fig. 2. As can be seen, nickel atoms are distributed unevenly over the diameter of the impurity microinclusion. In the central layer of microinclusion, the percentage of nickel atoms reaches $50 \%$, and toward the surface this value decreases by a spasmodic character. In the subsequent microinclusion layer, this value substantially decreases and amounts to $\sim 32 \%$. And on the surface layer, it decreases even more and equals to $\sim 25 \%$. As it turned out, a certain regularity is observed in the distribution of nickel atoms along the diameter of multilayer microinclusions $\left(\mathrm{d} \geq 10^{-6} \mathrm{~m}\right)$, according to which the percentage of impurity atoms increases towards the center of microinclusions and in this region it reaches its maximum value.
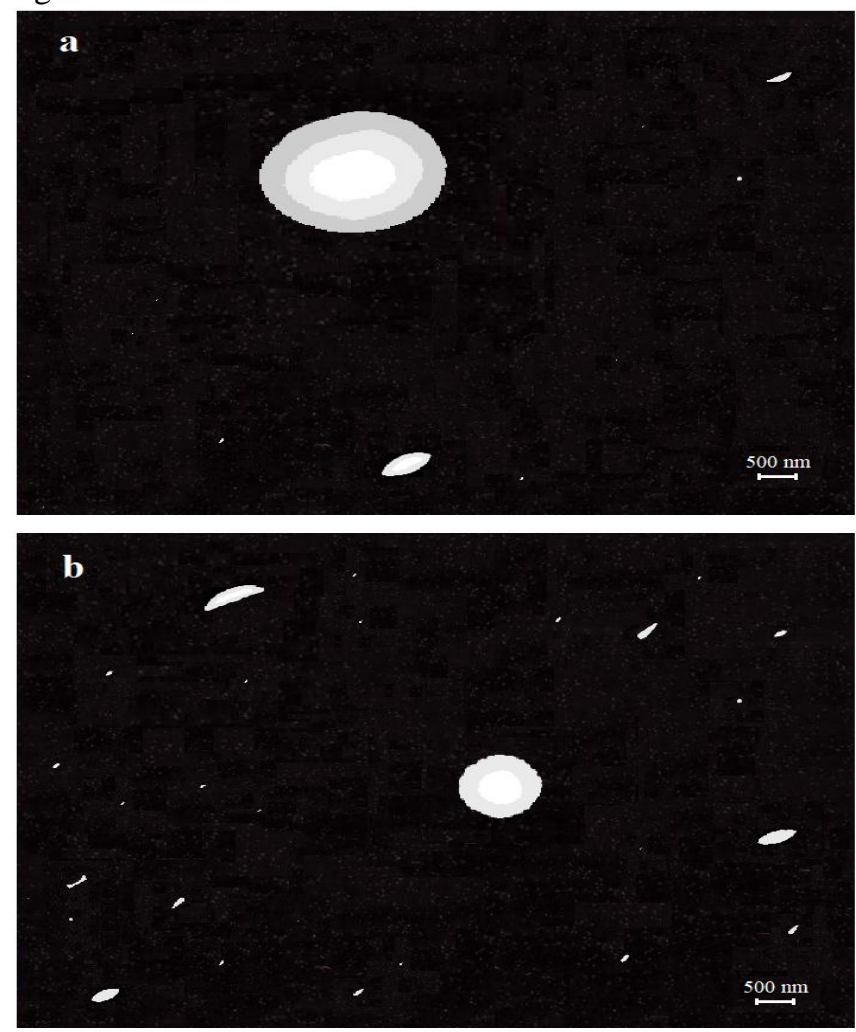

Fig. 1. Pictures of accumulations of nickel impurity atoms in $\mathrm{n}-\mathrm{Si}<\mathrm{Ni}>$ samples obtained with a slow (a) and fast (b) cooling rate.

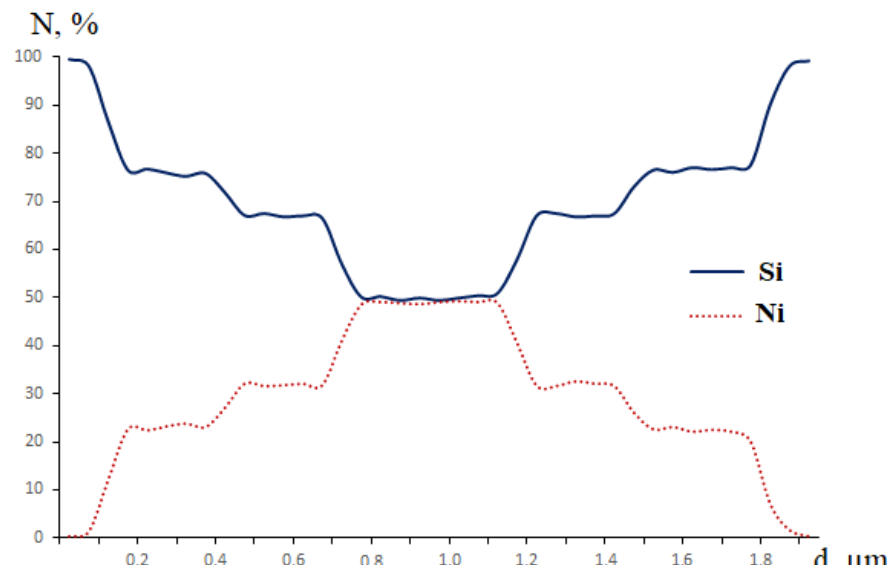

Fig. 2. The distribution of $\mathrm{Si}$ and $\mathrm{Ni}$ atoms over the diameter of the microinclusion, having a three-layer structure with a spherical shape, with a diameter $d=$ $1.9 \cdot 10^{-6} \mathrm{~m}$.

The obtained micrographs of $\mathrm{n}-\mathrm{Si}<\mathrm{Ni}>$ samples with rapid cooling showed that relatively small clusters of impurity atoms $(\mathrm{d} \leq 500 \mathrm{~nm}$ have a single-layer structure. The results of a quantitative analysis of nanoinclusions of a convex lenticular shape with a diameter of $600 \mathrm{~nm}$ are shown in Fig. 3. Graph of the distribution of nickel atoms the volume of this nanoinclusion shows that it has the same value over the entire diameter and amounts to 25\%. Analysis of the chemical composition of such impurity nanoinclusions confirms that nickel atoms are distributed in their volumes uniformly, i.e. they have a homogeneous structure (Fig. 3).

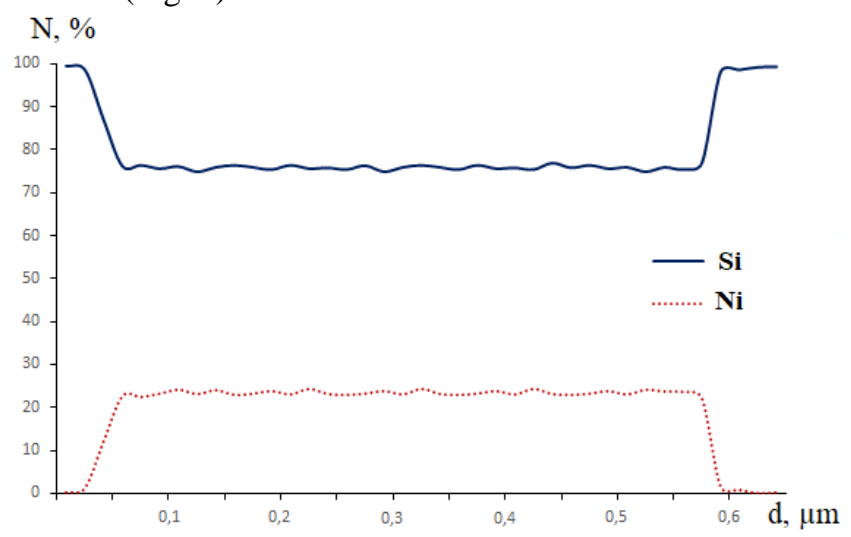

Fig. 3. Distribution of Si and Ni atoms over the diameter of the nanoinclusions, $d=600 \mathrm{~nm}$ in size with a lenticular shape.

As is known, at high temperatures of diffusion annealing, the atoms of technological impurities have a significant effect on the formation of microinclusions [8, 9]. Currently, when studying the mechanisms of formation of clusters of impurity atoms in the volume of semiconductor silicon, special attention is paid to the role of atoms of technological impurities in the process of their formation. Since the diffusion coefficient of atoms of technological impurities is much higher in comparison with the atoms of the main dopant, there are some assumptions that indicate the

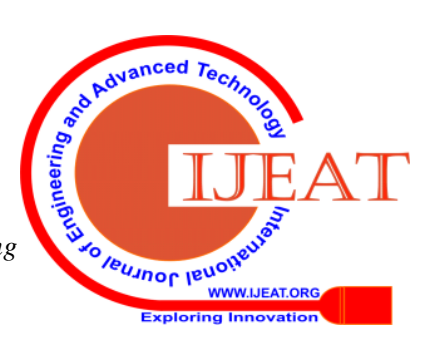


possible active participation of atoms of technological impurities in the formation of the structure of impurity clusters [10, 11].

The results of analyzes of the chemical composition of impurity microinclusions and nanoinclusions in $\mathrm{n}-\mathrm{Si}<\mathrm{Ni}>$ samples obtained both with fast and slow cooling showed that atoms of technological impurities such as $\mathrm{Fe}$ and $\mathrm{Cr}$ exist in their volume. The analyzes performed for a spherical microinclusion with a diameter of $\mathrm{d}=1.9 \cdot 10^{-6} \mathrm{~m}$, having a three-layer structure, made it possible to determine the quantitative indicators of atoms of technological impurities of iron and chromium in each shell of the microinclusion. The dependence of the percentage of atoms of uncontrolled impurities on the diameter of the microinclusion is shown in Fig. 4. As can be seen, the percentage of iron atoms in the central part of the microinclusion is $\sim 0.16 \%$, and for $\mathrm{Cr}$ atoms it is $\sim 0.12 \%$. In the subsequent layer of microinclusion, the percentage of $\mathrm{Fe}$ and $\mathrm{Cr}$ atoms decreases to $\sim 0.1 \%$ and $\sim 0.8 \%$, respectively. And in the surface layer, these values decrease to $\sim 0.07 \%$ and $\sim 0.04 \%$, respectively.

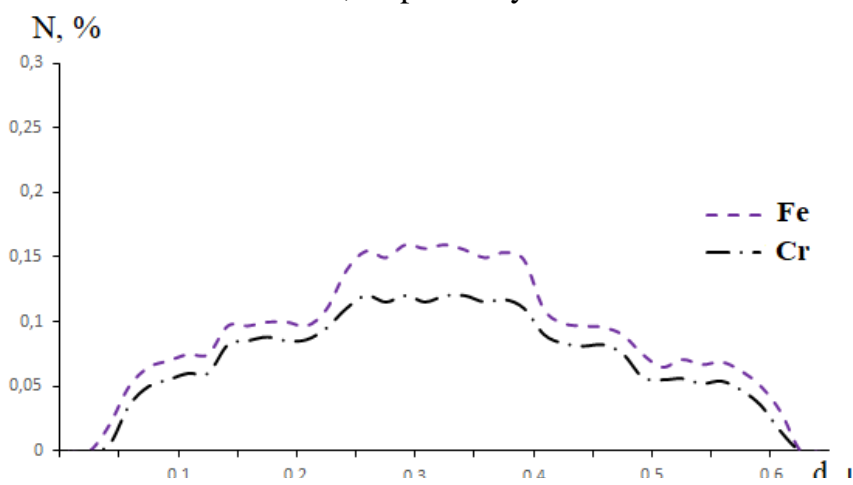

Fig. 4. The distribution of atoms of technological impurities $\mathrm{Fe}$ and $\mathrm{Cr}$ along the diameter of the microinclusion, having a three-layer structure with a spherical shape, with a diameter of $d=1.9 \cdot 10^{-6} \mathrm{~m}$.

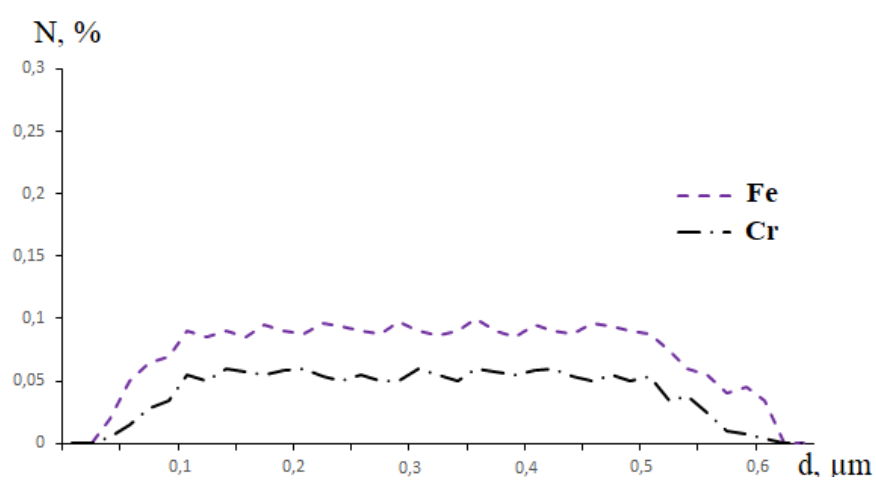

Fig. 5. The distribution of atoms of technological impurities $\mathrm{Fe}$ and $\mathrm{Cr}$ by the diameter of the shape.

In the course of studying the distribution of atoms of technological impurities over the volume of multilayer impurity clusters, it turned out that there is a certain regularity in their distribution over the diameter of microinclusions. In accordance with this pattern, the maximum percentage of atoms of technological impurities are located in their central part. In the direction from the nanoinclusions, $d=600 \mathrm{~nm}$ in size with a lenticular

center to the surface of impurity clusters, the percentage of atoms of these impurities decreases.

\section{CONCLUSION}

Thus, on the basis of the results obtained, it was revealed that with an increase in the $v_{\text {cool }}$ value, the probability of the formation of impurity nanoinclusions with relatively small sizes that have a needle-shaped, disk-like, lamellar and lenticular shape increases. At low values of $v_{\text {cool }} \leq 1 \mathrm{~K} / \mathrm{s}$, larger nanoinclusions are formed, which consist of several layers and have mainly a lenticular and spherical shape. It has been established that the number and distribution of impurity atoms over the volume of nanoinclusions depend on their size and shape. Morphological parameters of impurity nanoinclusions, i.e. the structural constructions, chemical composition, geometric shapes and sizes mainly depend on the cooling rate of the samples after diffusion annealing. In samples with a high cooling rate, after diffusion annealing, impurity nanoinclusions of various shapes are formed with relatively small sizes (up to $500 \mathrm{~nm}$ ) and they have a single-layer structure. At low cooling rates, lenticular and spherical nanoinclusions are formed with sizes up to $\sim 900 \mathrm{~nm}$, which have a multilayer structure. It was established that in the volume of impurity nanoinclusions there are atoms of technological impurities like $\mathrm{Fe}$ and $\mathrm{Cr}$. It turned out that a certain regularity is observed in the distribution of atoms of technological impurities over the diameter of nanoinclusions, according to which the percentage of atoms of these impurities decreases in the direction from the center to the surface of the nanoinclusions.

\section{REFERENCES}

1. Yoshikawa T, Morita K, Kawanishi S, Tanaka T. Thermodynamics of impurity elements in solid silicon // Journal of Alloys and Compounds. 2010. Volume 490. Issues 1-2. pp. 31-41.

2. Fistul V.I. Atoms of dopants in semiconductors. - M .: 2004. -432 p.

3. Talanin V.I., Talanin I.E. Diffusion model of the formation of growth microdefects: a new approach to defect formation in crystals // FTT. 2016. Volume 58. Issue. 3, pp. 417-427.

4. Buonassisi T., Heuer M., Istratov A.A., Pickett M.D., Marcus M.A., Lai B., Cai Z., Heald S.M., Weber E.R. Transition metal coprecipitation mechanisms in silicon // Acta Materialia. 2007. Vol. 55. pp. 6119-6126.

5. Turgunov N.A. Formation of Cobalt Impurity Microinclusions in Silicon Single Crystals // Inorganic Materials, 2018, Vol. 54, No. 12, pp. 1183-1186.

6. Turgunov N.A. Investigation of impurity nanoinclusions of cobalt in monocrystals of silicon // Science and World. 2018. No. 5 (57), pp. 21 24.

7. Turgunov N.A. Study of the structure and chemical composition of microinclusions of cobalt impurities in silicon single crystals // Uzbek Physical Journal. 2016. Vol. 18. No. 5. pp. 316-321.

8. Astashenkov A.S., Brinkevich D.I., Petrov V.V. Properties of silicon doped with nickel impurity by diffusion // Reports of BSUIR. 2008. No. 8 (38). pp. 37-42.

9. Talanin V.I., Talanin I.E. The use of the diffusion model of the formation of growth microdefects to describe defect formation in heattreated silicon single crystals // FTT. 2013. Volume 55. Issue. 2, pp. 247-251.

10. Klimanov Evgeny Alekseevich Formation of thermal defects in silicon grown by crucible-free zone melting // Journal: Successes in Applied Physics. Volume: 4. No. 5. 2016. pp. 471-479.
Sciences Publication

(C) Copyright: All rights reserved. 
11. Kritskaya T.V., Panchenko O.V. Investigation of the doping processes of dislocation-free silicon single crystals grown by the Czochralski method // News of Higher Education Institutions. Materials of electronic equipment. 2008. No. 1. pp. 4-8.

\section{AUTHORS PROFILE}

Turgunov Nozimjon Abdumannopovich is also the scientific secretary of the Research Institute of Semiconductor Physics and Microelectronics at the National University of Uzbekistan, E-mail: tna_1975@mail.ru

Berkinov Elmurod Khoshimjonovich is also a doctoral student at the Research Institute of Semiconductor Physics and Microelectronics at the National University of Uzbekistan. 